\title{
A Graph-Based Approach for Aspect Extraction from Online Customer Reviews
}

Rakesh Kumar, Aditi Sharan

Jawaharlal Nehru University, New Delhi

India

\{rakesh.kmr2509@gmail.com\}\{aditisharan@gmail.com\}

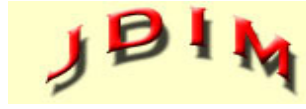

Journal of Digital

Information Management
ABSTRACT: E-commerce websites have become main market players in the 21 st century due to advancement in the internet technology. Apart from buying products online, customers are also providing reviews on the products purchased by them. These reviews help new customers to buy various products according to their needs, liking, and preferences. However, millions of reviews are added by the customer on a daily basis. To extract meaningful information manually from these huge amounts of reviews is a tough task. So, it is required to develop an automatic analytics tool for the review sentences. Aspect extraction is one of the vital tasks in the process of meaningful information extraction from the products having various entities. In this work, a novel product aspect extraction approach has been proposed which utilize a graphbased technique with the integration of statistical and semantic information. The analysis of experimental results shows that the proposed approach is efficient and effective in comparison to the state of art methods.

Subject Categories and Descriptors

[H.2.8 Database Applications]: Data mining; [K.4.4 Electronic Commerce]

General Terms: E-Commerce, Customer Reviews, Opinion mining

Keywords: Opinion Mining, Feature Extraction, PageRank Algorithm, Online Product Reviews

Received: 13 November 2019, Revised 17 March 2020, Accepted 3 April 2020
Review Metrics: Review Scale: 0/6, Review Score: 4.98, Interreviewer Consistency: 85.1

DOI: $10.6025 / \mathrm{jdim} / 2020 / 18 / 3 / 99-108$

\section{Introduction}

In the recent decade, since most of the content on the internet is user-generated, so most of the products are reviewed by the users. Almost all shopping websites, blogs, or forums allow the customers to post their ratings and opinion about the products or services. The example for the same is www.amazon.com, rottentomatoes.com, epinions.com. The virtual world has a lot of influence on the customers as well as it provides a lot of information on business intelligence and marketing. Most of the reviews are considered as ratings and feedback which helps the customers to decide on which product to buy and helps the retailer and manufacturers to understand the positives and negatives of the product. Nowadays everyone posts their reviews, so the number of reviews also increases and it is difficult for the new user to get an overall picture of all the opinions of the customers who have used the product. It s very difficult to do it through manual analysis. Therefore, it is required to develop proper analysis and summarization tools for the review's analysis which provides a better idea about the product to new customers.

To extract meaningful information from these huge amount of online review various techniques have been suggested in the literature $[1,27,32,19,18,4,3,30,11,5,36]$. In modern 
days, online marketing and e-commerce have a great impact on the economy of every country [12]. These ecommerce websites are providing facility to their customers to provide valuable feedback which helps new customers to buy products according to their preferences and liking. The review analysis also provides better feedback to the manufacturers which helps to enhance the product quality.

Information overloading and different preferences are two major problems that hinder customers from taking advantage of product reviews. One has to go through millions of reviews to decide the goodness of a product which is known as information overloading [24]. Information overloading influences the decision of the customers in a negative way by producing fewer customer purchases [17, 34 , 2]. Although the text mining techniques have been applied to online review analysis, these techniques mainly used for identifying general trends or certain patterns of online consumer reviews instead of solving the individual selection or recommending a specific product or analyzing a particular solution. For example, if one has to buy a mobile phone, some opt for the appearance, or the functions, or for the brand and some customers go for the price that may work as the deciding factor. The majority of the online forums do not consider the preference of the individual customers. Only a few of them have personalized and the product featured reviews. For the users, it is impossible to go through all reviews to extract specific information about a product. To deal with this problem, we have proposed a framework that automates the product aspects extraction process from online customer reviews. This framework is based on a graph-based approach that integrates statistical and semantic information to identify the important aspects of customer review. To illustrate the effectiveness of the proposed approach the experiments have been performed with benchmark data sets. The experimental results show that the proposed method is effective and efficient in comparison to the state of art methods.

The rest of the paper is organized as follows: Section 2 presents the related research on product feature extraction. In section 3 we present the proposed framework for aspect extraction using a graph-based approach. Section 4, contains experiments and analysis. Finally, we conclude the paper with a summary and directions for future work in Section 5.

\section{Related Work}

Feature extraction plays a vital role in the aspect based review analysis of the products. A lot of studies have been carried out on feature extraction which has achieved directed goals. In the description of the products, mostly nouns and adjective phrases are utilized in the review sentences. Hu and Liu [18] proposed a method based on association rule mining to discover nouns terms with high frequency as features. Popescu and Etzioni [20] proposed an extended version of $\mathrm{Hu}$ and Liu [18] method to rank aspect based features with a new measure PMI-IR (Pointwise Mutual Information and Information Retrieval) for semantic resemblances of the features and product aspects. Li et al. [15] proposed an enhanced method based on the extended PMI to increase the efficiency of the feature extraction methods for the review sentences. Long et al. [16] developed a new method that firstly extracts the nouns terms with greater frequency as basic features and after that finds other features based on informative dissimilarity between basic nouns features and other terms.

The experimental study of Scaffidi et al. [28] shows that frequent terms in the product review sentences have a high possibility to be as features. Part-of-Speech tagging is used in frequency-based methods, which has high accuracy. This approach ignores the features, which are less frequent but are important. One of the examples for the same is the features of phones that are quality of sound and power. Thus, only using the frequency-based method is not enough. Zhuang et al. [38] developed a method to extract features for the analysis of movie reviews utilizing four dependency relations occurring with a high frequency known as subject-predicate relations, adjectival modifying relations, relative clause modifying relations, and verb-object relations. Wu et al. [33] proposed another dependency relation-based method by considering nouns terms and verb terms as core features and adjectives related to a particular noun as sentiment terms. Poria et al. [39] proposed a rule-based approach that exploits common-sense knowledge and sentence dependency trees to detect both explicit and implicit aspects.

A method based on the direct and indirect relations has been proposed by Qiu et al. [23] named as a double propagation algorithm (DP). In another work, the DP algorithm has been modified by utilizing the seed opinion lexicon. Another version of the DP algorithm proposed by Zhang and Zhou [14] integrating indirect dependency with comparative constructions for discovering subjective based features.

Zhang et al. [37] shown that apart from a direct relationship there may also exist a Part-whole relationship in the terms of the review sentences. The proposed by Zhang et al. utilizes direct relations and the part-whole relations named phrase pattern, sentence pattern, and "no" pattern to extract features from the review sentences. Another version of the method has been proposed by $\mathrm{Su}$ and Lynn [29] which uses only phrase patterns to discover features from the review sentences. The features related to the domain-dependent and independent views have been extracted by a Two-fold rules-based method which is developed by Rana and Cheah [25]. The dependency trees may play a vital role to discover various features from the review sentences. Poria et al. [21] proposed a method to discover features utilizing a dependency parse tree combining with knowledge-based rules. This method can discover features having a lower frequency from the large set of text documents. Feature ranking 
may play a vital role in the extraction core set of features from review sentences. Rana \& Cheah [40] had proposed a rule based hybrid approach that exploits sequence pattern and normalized Google distance to extract explicit as well as implicit aspects.

The overall sentiment score of a product cannot be used to filter the candidate features and sentiment terms. The individual feature of a product has a great influence on the decision-making process of the customers. So, it is required to develop automatic tools to rank the different features of a product efficiently. The noun phrases that are associated highly with sentiments terms have a greater possibility to be the core product features. Eirinaki et al. [6] developed a High Adjective Count (HAC) method which calculates an opinion score for noun phrases to select basic features of the products. Yan et al. [35] and Zhang et al. [37] used similar ideas with HITs and Page rank technique to determine the ranking of the core candidate features of the products. In this work, the page rank algorithm has been integrated with candidate pair extraction and filtering for efficient aspect extraction. Gunjan et al. [41] used the graph based semi-supervised learning approach for aspect term classification.

\section{Proposed Methodology}

The users prefer products based on different aspects of these products. The liking of products based on aspects makes aspect extraction vital components in the sentiment analysis. Aspect extraction is a challenging task due to the existence of various complexity in the review data sets. In this section, we have proposed a graphbased approach that integrates statistical and semantic information to identify the important aspects of customer review. The proposed framework mainly contains three modules namely pre-processing, pattern extraction, and graph-based aspect ranker. Based on the framework, two variants named as graph-based aspect extraction (GBAE) and graph-based aspect extraction with filtering (GBAEF) have been proposed. Section 3.1 contains the detailed description proposed GBAE method and section 3.2 contains the detail of the proposed GBAEF method.

\subsection{Proposed GBAE Approach}

The proposed model based on the three modules- Preprocessor, Pattern Extractor, and Graph-Based Aspect Ranker. Figure 1 contains the framework of the proposed GBAE approach. The module is the preprocessor which takes set reviews as input and applies necessary preprocessing techniques to produce data sets for further processing. Then after pattern extractor module returns patterns that are (Aspect-Sentiment) word pairs. The next module in the proposed approach is graph-based Aspect ranker which constructs a graph by treating Aspects/Sentiment words as nodes. The edges in the constructed graph represent a relation between aspects and sentiments pairs. Once the graph is constructed aspect based ranker module generates score/weight to the aspects of the graph and sorts these aspects on weights. The top aspects provide the output of our systems. Following are detail description of each module utilized in the GBAE approach:

\subsubsection{Preprocessing (Part-of-Speech Tagging and Stemming)}

The preprocessor accepts reviews text as input and then apply part of speech (POS) tagging and stemming to input received. The POS tagger module maps each word of the input text file to a particular part of speech like noun, adjective, verb, etc. Stemming is the process of providing the base or stems word for a given set of words. In this work, the software of Stanford has been utilized for POS tagging and stemming [13].

\subsubsection{Candidate Pair Extraction}

Candidate pair extraction is the first step of the pattern extraction module. In this step, we use a dependency relation-based approach to extracts product aspects based on dependency relations among terms that appeared in review sentences.

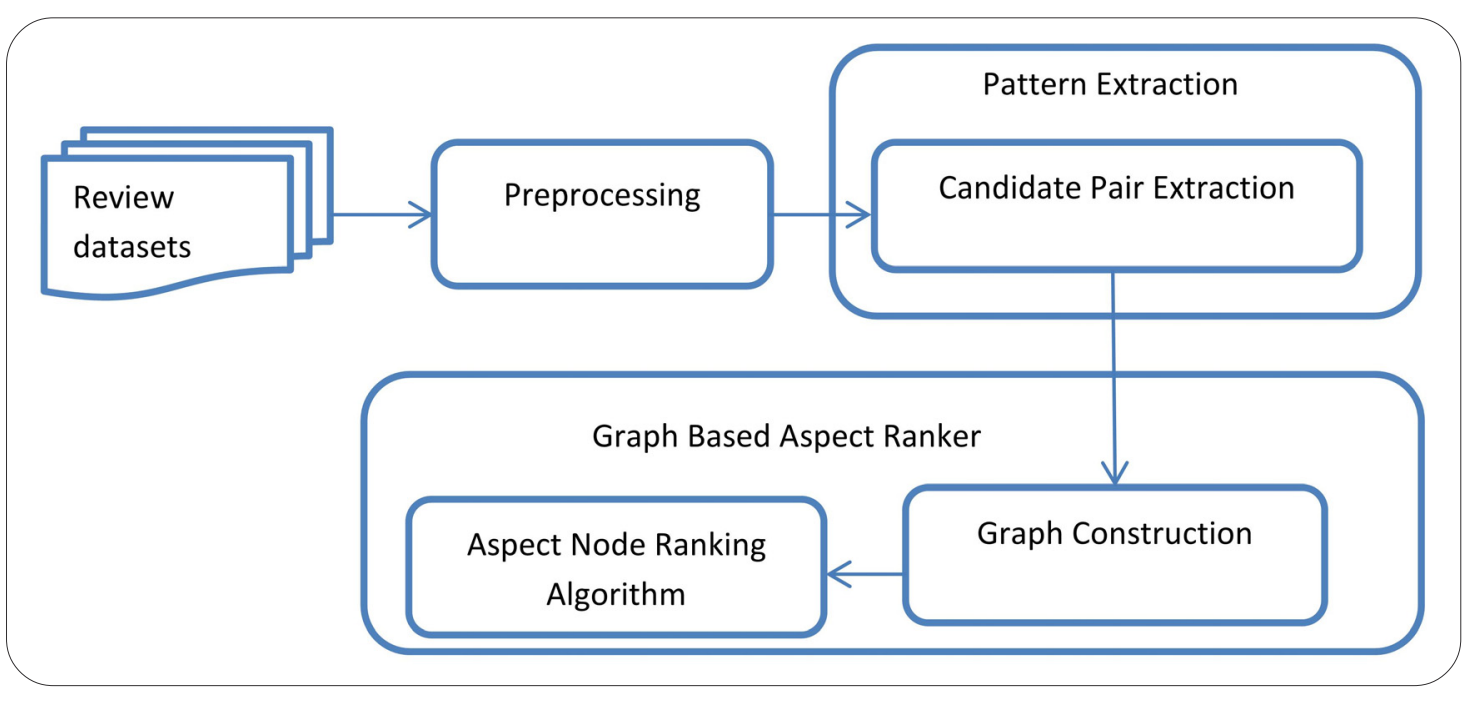

Figure 1. Framework for proposed system graph-based aspect extraction without filtering (GBAE) 


\section{nsubj advmod She looks very beautiful}

Figure 2. The syntactic structure of a sentence consisting of dependencies

Various studies in the area of review analysis show that there exist co-relation between feature terms and words related to sentiments. This co-relation may be utilized to extract various product features from review sentences [7]. This approach firstly finds the dependency relations from the review sentences and then extract product aspects from the various dependency relations.

An asymmetric binary association between head/governer and modifier/dependent is known as dependency relation. The dependency syntax explains the structures of the sentences with a set of dependency relations [8]. Figure 2 contains a sentence that has a dependency relation. In the given sentence the head 'She' and a modifier 'looks' make a dependency relationship 'nsubj' and the head 'very' and a modifier 'beautiful' makes other relationship 'avdmod'.

A dependency syntax analysis may help to discover the term s role and dependency relations in a review sentence. Stanford Parser has been utilized in our study to discover features of the candidate product and related sentient term (http://nlp.stanford.edu).

A given sentence may contain various dependency relations but only a few relations have been utilized to discover features of products [33]. In this study, four types of dependency relations (nsubj : subject-predicate relations, amod : adjectival modifying relations, rcmod: relative clause modifying relations and dobj: verb-object relations) have been utilized for the extraction of product features. The example shown in Figure 2 contains two dependency relationships: 'she' and 'looks' makes the 'nsubj' relationship; and 'very' and 'beautiful' makes the 'avdmod' relationship. The proposed method selects only one dependency relationship of 'nsubj', like (she, OK) which will act as pairs for candidate feature-sentiment pairs.

In this approach, four dependency relations have been selected from the various dependency relations extracted from the Stanford Parser. These four dependency relations have been utilized to create a set of candidate aspect-sentiment pairs from the given review documents. It can be observed that some irrelevant pair also appears in the extracted aspect-sentiment pair. The filtering approach is therefore required to deal with this problem.

\subsubsection{Graph-based Aspect Ranker}

In this module, firstly we construct an aspect-sentiment relation network based on extracted aspect-sentiment pairs and then apply the AspectNodeRanking algorithm to rank aspect nodes. This module is divided into two steps namely Graph construction and the AspectNodeRanking algorithm.

\subsubsection{Graph Construction/Representation}

In our proposed method, graph construction is inspired by the page rank algorithm. Page rank is one of the wellknown algorithms to rank the websites in a highly effective manner [26,31]. It considers that the ranking of a particular website also depends on the other websites linked by it [9]. The variability of the importance of websites is done by Page Rank. The page rank algorithm provides equal importance to all other websites linked by that website. Lets there are $\mathrm{N}$ websites, then a vector $P=(p$ $(1), p(2) \ldots p(N))$ provides the ranking value of websites. The adjacency relationship among various websites is described by a coefficient matrix A as follows:

$$
A_{i j}=\left\{\frac{1 / o_{i}, \text { if } O_{i}>0,(i, j) \in E}{1 / N, \text { if } O_{i}=0}\right\}
$$

Where $E$ denotes the set of directional links among various websites; $O_{i}$ denotes the number of weblinks to a particular website $i$. Then, the vector $P$ can be described as follows:

$$
P=\left((1-\propto) \frac{1}{N}+\propto A^{T}\right) P
$$

Where $\propto$ denotes a damping coefficient. The Page rank measure may be determined by using the power iteration method [10].

In aspect extraction, the aspect term is likely to be a noun or a noun phrase in the product review even if it is modified by multiple adjectives since many tend to use adjectives to describe their views and opinion of the product. [6][5]. Similarly, if the adjective is modified as multiple product aspect terms it is likely to be a sentiment. Thus a product aspect and its sentiment word both can be treated as a network node, and modifying relations between the candidate aspect and sentiment words can be treated as network edges. This kind of network is called as an aspect-sentiment relation network.

\subsubsection{Aspect Node Ranking Algorithm}

After graph construction described by the above procedure, the aspect-sentiment relation network and rank of the aspect nodes have been derived. The occurrence of a noun phrase in a review makes a high possibility to be 
product aspects. We define the importance $p(i)$ of a network node $i$ as follows:

$$
p(i)=(1-\propto) H(i)+\propto \Sigma_{(j, i) \in E} \frac{p(j)}{O_{j}}
$$

where $\propto$ denotes a damping coefficient; $p(j)$ denotes the importance score of the node $j ; O$ denotes the number of linked nodes to $j$, and $E$ denotes the set of an edge in an aspect-sentiment relation network; $H(i)$ denotes the frequency occurrence function of node $I$ and it is defined as:

$$
H(i)=\frac{N \ln f(i)}{\operatorname{In}\left(\prod^{N}{ }_{j=1} f(j)\right)}
$$

Where $N$ denotes the number of nodes in the graph; $f(i)$ denotes the occurrence frequency of node $i$ in an entire review corpus. The integration of log function may reduce the high occurrence frequency adverse effect of less relevant term in the node ranking. The vector $P=(p(1), p(2)$, $p(3), \ldots, p(N))^{T}$ may be determined by utilizing a power iterative method. After extraction of aspect-sentiment pairs, the aspect-sentiment relation graph will be constructed. The AspectNodeRank scores of each aspect node will be determined by the AspectNodeRank algorithm. The AspectNodeRank scores of aspect nodes greater than a specified threshold will be selected in the product aspect list.

\subsection{Proposed GBAFE Approach}

In this section, a modified version of the GBSE has been proposed which integrates a filtering criterion in the pattern extraction module. This framework is also based on three modules- Pre-processor, Pattern Extractor, and Graph-Based Aspect Ranker. The framework for the proposed GBAFE approach is shown in Figure 3. In this variant, one sub-module has been added called as filtering module in the pattern extraction module. The working of the proposed GBFE framework will be similar to GBSE except for the filtering sub-module. The added filtering sub-module drop the irrelevant pairs appear in the extracted aspect-sentiment pair. The following sub-section elaborates the working of filtering approach used in the proposed GBAEF framework:

\subsubsection{Filtering}

Filtering is the sub-module of the pattern extraction module. In this step of the pattern extraction module, we focus on filtering some irrelevant aspects-sentiment pairs from the candidate pairs by using a statistical approach: AS-score. To explain the AS-score measure, it is helpful to first introduce PMI.

\section{Point-wise Mutual Information (PMI)}

$\mathrm{PMI}$ is the widely used parameter to capture association among different entities. In statistics, PMI captures the mutual dependence of two random variables. PMI has wide applications in many areas like in text mining it is used to capture the occurrence of two words together. PMI can be used to measure the probability association between two words [22]. Let $X$ and $Y$ are the two different words in a text document then PMI can be calculated in the following way:

$$
P M I(a, b)=\log \frac{P(a, b)}{P(a) P(b)}
$$

Where $P(X)$ and $P(Y)$ are the probability of occurring $X$ and $Y$ word in the document, $P(X, Y)$ is the occurrence of word $X$ and $Y$ together in the given document. The probability $P(X), P(Y)$, and $P(X, Y)$ usually calculated by counting the number of times words $X$ and $Y$ occurring in the text document with normalization. In the same way, $P(X, Y)$ is measured by counting co-occurrence $X$ and $Y$ in the given text document. The PMI measurement algorithm usually provides optimal value for the large size of the text document.

\section{AS-score}

In this approach, we have introduced a new measure named AS-score which is based on PMI. The AS-score

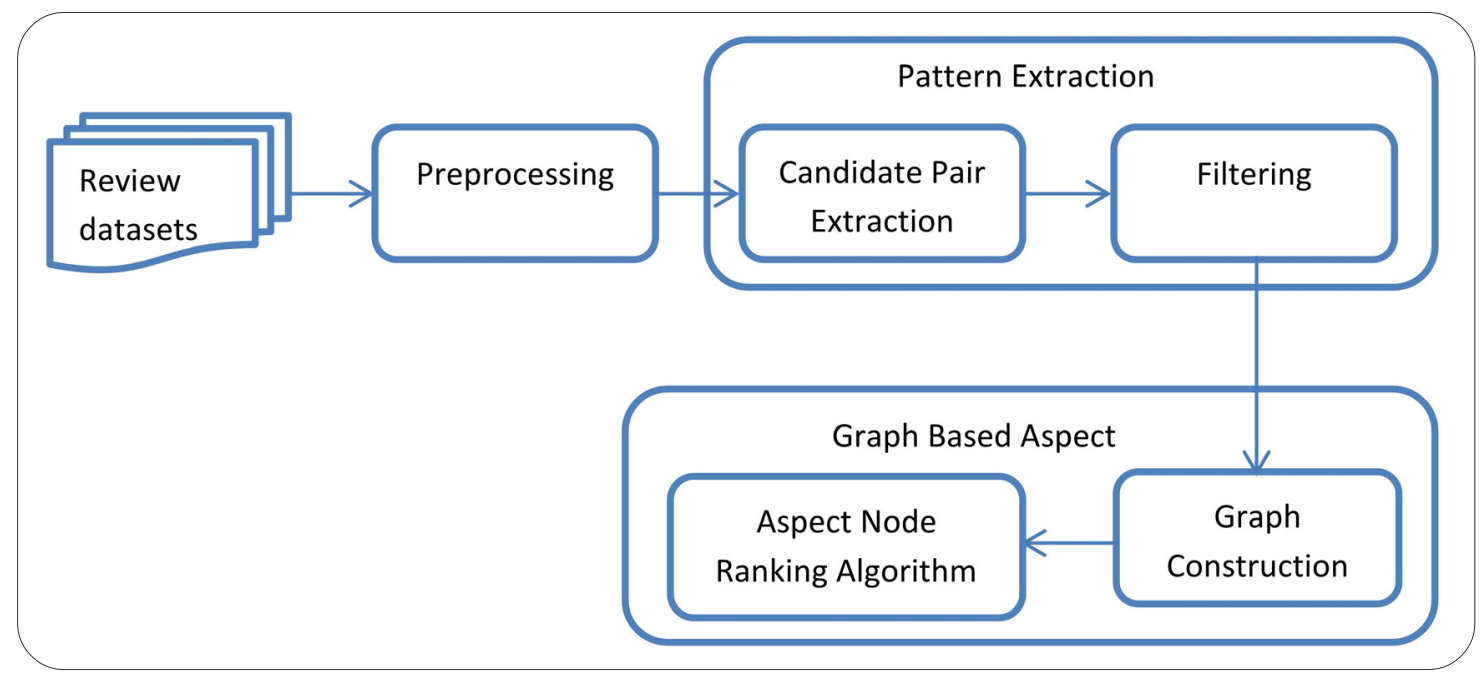

Figure 3. Framework for proposed system graph-based aspect extraction with filtering (GBAEF) 
uses both inter-relation information between words and frequency of words to measure the score. It scores each candidate aspect-sentiment pairs with the AS-score metric. The AS-score is defined as follows:

$$
A S-\operatorname{Score}(x, y)=f(x) * \log _{2}([f(x, y) / f(x) * f(y)])
$$

Where $x$ denotes the aspect word, $y$ denotes the sentiment word, $f(x)$ denotes the frequency of the review sentences in the text document which contains the aspect word $x, f(x, y)$ denotes the number co-occurrence of the aspect word $x$ and sentiment word $y$ for a sentence in the given text document.

Apart from mutual information between an aspect and a sentiment word; the AS-score measure also considers the frequency of each aspect. The integration of add-one smoothing to the measure removes the possibility of being zeros to all co-frequencies. The AS-score measure filters the less relevant candidate aspect-sentiment pairs.

\section{Experimental Results}

In this section, the experimental results for the proposed model have been discussed. To show the effectiveness of the proposed model, the experimental results have been determined in the first steps, then after a comparative study has been done with benchmark results. The following are the description of the data set, the Design of Experiments, Experimental results, and analysis.

\subsection{Dataset Description}

To evaluate the performances of the proposed method customer reviews data sets of five products have been utilized $[19,7]$. The data set related to electronics products covering various domains namely Canon G3, Apex AD2600 Progressive-scan DVD player, Nikon Coolpix 4300 , Creative Labs Nomad Jukebox Zen Xtra 40 GB, and Nokia 6610. These data sets have been widely used in literature to evaluate performances of opinion mining techniques. Table 1 contains the descriptions of the datasets like the number of reviews, number of review sentences, and number manually annotated aspects. The data sets used in the experiments are annotated by human annotators and these annotated product features form gold standards that s why directly used as a reference for each dataset.

\subsection{Design of Experiments}

The experiments were performed on 5 product review datasets in which each product review dataset contain text review and their aspects. In the experiment, the label aspects have been considered as the benchmark for result evaluation.

The proposed framework based on the three modulesPre-processor, Pattern Extractor, and Graph-Based Aspect Ranker. The input to preprocessor is the review dataset after preprocessor pattern extractor module returns patterns that are (Aspect-Sentiment) words pairs. Then in the graph-based ranker module, a graph is constructed, where nodes representAspects/Sentiment words and edges represents a relation between aspects and sentiments pairs. Once the graph is constructed aspect based ranker module generates score/weight to the aspects of the graph and sorts these aspects based on weights. For the GBAE approach, the experiment without a filtering method has been performed. A filtering approach has been utilized in the GBAEF method for aspect extraction. In the first step, all the aspect-sentiment word pairs have been considered extracted from the dependency parser and then applied graph-based analysis for aspect extraction. The AS-Score have been calculated for all the aspect-sentiment word pairs extracted in the previous step and the threshold value for different data set have been determined through experiment. Based on the threshold value, dependency pairs are filtered out. Now, these dependency pairs have been used for graph-based analysis.

\subsection{Experimental Results and Analysis}

In this section, the proposed approach has been applied to 5 product review datasets. At the same time, we compare the results of the main steps of the proposed model on the Recall measure. To show the effectiveness of the proposed method, the experimental result has been compared with the state of art methods. In [19] authors have developed an association rule mining based approach named ARM for expect extraction. Wei et al. developed a method named as SPE based on the semantic relationship for the aspect extraction of the product [5].

\begin{tabular}{|l|l|l|l|l|}
\hline Dataset & $\begin{array}{l}\text { Number of } \\
\text { reviews }\end{array}$ & $\begin{array}{l}\text { Number of review } \\
\text { sentences }\end{array}$ & $\begin{array}{l}\text { Number of manual } \\
\text { aspects }\end{array}$ & $\begin{array}{l}\text { Number of Single- } \\
\text { word aspect }\end{array}$ \\
\hline Canon G3 (D1) & 45 & 597 & 100 & 65 \\
\hline Nikon Coolpix 4300 (D2) & 34 & 346 & 74 & 38 \\
\hline Nokia 6610 (D3) & 41 & 546 & 109 & 74 \\
\hline $\begin{array}{l}\text { Creative Labs Nomad Jukebox } \\
\text { Zen Xtra (D4) }\end{array}$ & 95 & 1716 & 180 & 120 \\
\hline Apex AD2600 (D5) & 99 & 740 & 110 & 71 \\
\hline
\end{tabular}

Table 1. Summary of customer review dataset 


\begin{tabular}{|l|l|l|l|l|l|}
\hline Product & ARM & SPE & Bagheri & GBAE & GBAEF \\
\hline D1 & 63 & 75 & 70.7 & 95.384615 & 87.692308 \\
\hline D2 & 67.6 & 75.7 & 68.6 & 89.473684 & 81.578947 \\
\hline D3 & 57.8 & 72.5 & 71 & 85.135135 & 81.081081 \\
\hline D4 & 56.1 & 65 & 57 & 87.5 & 78.333333 \\
\hline D5 & 60 & 70 & 65 & 85.915493 & 81.690141 \\
\hline
\end{tabular}

Table 2. Recall values of the ARM, SPE, Bagheri, GBAE and GBAEF model on Five Data set

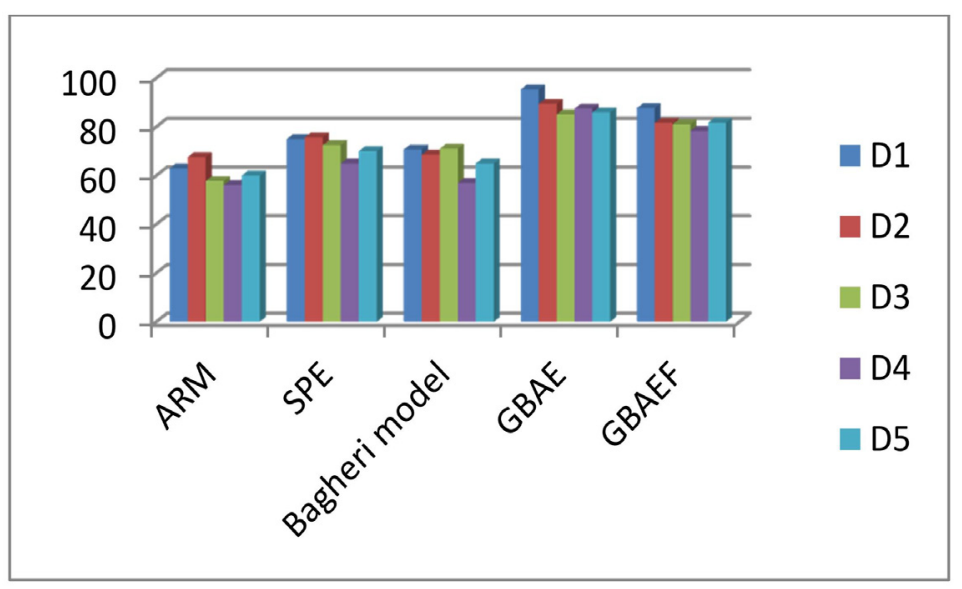

Figure 4. Bar graph for comparative study for recall

\begin{tabular}{|l|l|l|l|l|}
\hline Dataset & No. of Aspects & No. of Nodes & No. of Edges & Recall \\
\hline D1 & 62 & 942 & 1042 & 95.384615 \\
\hline D2 & 34 & 597 & 603 & 89.473684 \\
\hline D3 & 63 & 789 & 754 & 85.135135 \\
\hline D4 & 105 & 1712 & 2374 & 87.5 \\
\hline D5 & 61 & 834 & 980 & 85.915493 \\
\hline
\end{tabular}

Table 3. Recall value of the proposed method GBAE

Table 2 contains the experimental results of the proposed method with SPE and ARM techniques for the customer review datasets. The minimum support threshold set as $1 \%$ for frequent aspect identification for the ARM and SPE technique. Table2 shows the recall values for ARM, SPE, Bagheri, GBAE, and GBAEF methods for all five data sets. The analysis of the recall values shown in Table 2 indicates that the proposed GBAE method has produced almost $20 \%$ for all data sets in comparison to ARM, SPE, and Bagheri models. The analysis also shows that the proposed method GBAEF has produced better recall values for all data sets in comparison to ARM, SPE, and Bagheri models. Further analysis of the experiment shows that the recall value of the GBAE is slightly higher than the GBAEF method. The overall analysis of experimental results shown in Table 2 and Figure 4 indicates that GBAE and GBAEF have the capability to produce better results.
Table 3 contains the recall values for GBAE method with respect to the number of aspects, number of nodes, and number of edges. In the same way Table 4 contains the recall values for GBAEF method with respect to number of aspects, number of nodes, and number of edges. Figure 5 contains the comparative study of the number of nodes and edges by applying filtering and without filtering approach. The analysis shows that by reducing the number of edges and nodes through the filtering approach, the recall values have not reduced much but the size of the graph has been reduced drastically. So if the data set is bigger then there is a high possibility that irrelevant nodes and edges may get added in the graph. The proposed method with filtering technique (GBAEF) may reduce the number of irrelevant nodes and edges for graph construction. Reduction in the graph size reduces the time complexity of the method. The comparative study of 


\begin{tabular}{|l|l|l|l|l|l|}
\hline Dataset & AS-score (threshold value) & No. of Aspects & No. of Nodes & No. of Edges & Recall \\
\hline D1 & 0.0003 & 57 & 697 & 865 & 87.692308 \\
\hline D2 & 0.0005 & 31 & 461 & 501 & 81.578947 \\
\hline D3 & 0.0004 & 60 & 600 & 617 & 81.081081 \\
\hline D4 & 0.0003 & 94 & 1160 & 1856 & 78.333333 \\
\hline D5 & 0.0005 & 58 & 599 & 771 & 81.690141 \\
\hline
\end{tabular}

Table 4. Recall for the proposed method GBAEF with AS-score filtering

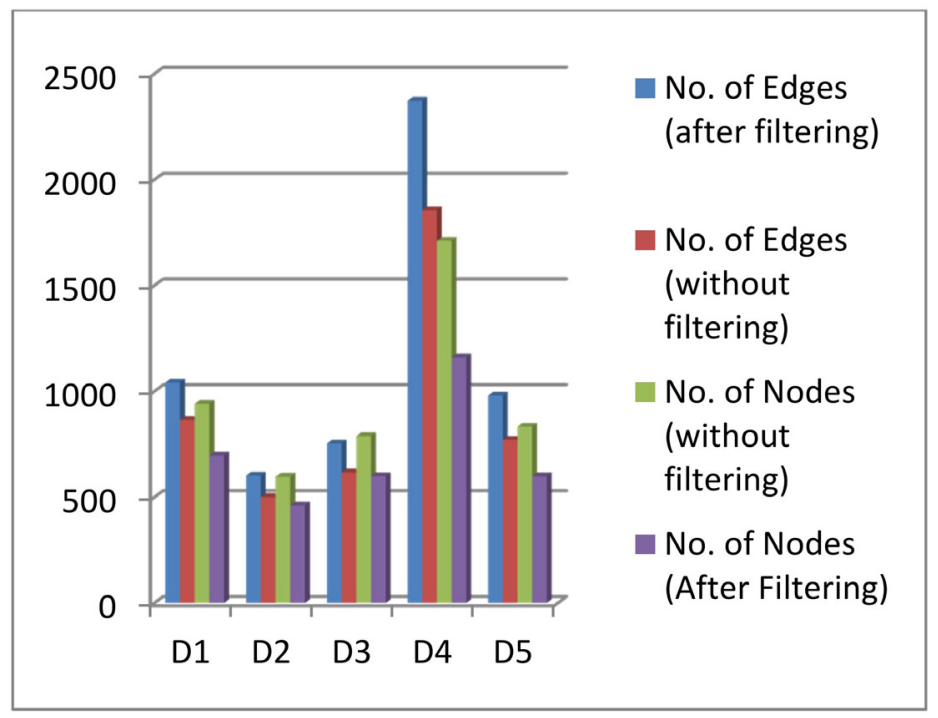

Figure 5. Bar graph of graph analysis before and after filtering

Table 3 and 4 indicates that for the same data sets when filtering is applied with different threshold value the number of nodes and edges have been reduced with high margin. For example, the numbers of nodes are 942 for the D1 data set with the GBAE method, but after applying the filtering method (GBAEF) with threshold value 0.0003 the number of nodes reduced to 697 . Figure 5 indicates a comparative study in the number of edges and nodes for all data sets with filtering and without filtering methods. The experimental results conclude that the proposed approach may achieve the same level of recall values while reducing the number of nodes and edges of the graph.

\section{Conclusion}

This paper presents a novel graph-based approach to extract aspects from the set of reviews sentences. The method mainly contains three modules named as a preprocessor, pattern extractor, and graph-based aspect ranker. The main contribution has been made into pattern extractor and graph-based aspect ranker modules. The pattern extractor modules use the dependency-based technique to extract products from the preprocessed review documents. A new measure named AS-score to rank features for filtering technique is proposed which selects relevant aspect sentiment pairs. The page rank algorithm has been utilized for graph-based aspect ranking. The experiment results with benchmark data sets show that the proposed approach has the capability to produce better performances in compassion to state of art methods.

\section{References}

[1] Balahur, A., Hermida, J. M., Montoyo, A. (2012). Detecting implicit expressions of emotion in text: a comparative analysis, Decision Support Systems, 53, 742753.

[2] Ghose, A., Ipeirotis, P. G., Li, B. B. (2014). Examining the impact of ranking on consumer behavior and search engine revenue, Manag. Sci. 60 (7) 1632-1654.

[3] Liu, B., Hu, M., Cheng, J. (2005). Opinion observer: analyzing and comparing opinions on the web, In: Proceedings of International Conference of World Wide Web, Publishing, 2005, p. 342-351.

[4] Lin, C., He, Y., Everson, R., Ruger, S. (2012). Weakly supervised joint sentiment-topic detection from text, IEEE Transactions on Knowledge and Data Engineering 24 (6) 1134-1145.

[5] Wei, C. P., Chen, Y. M., Yang, C. S., Yang, C. C. (2010). Understanding what concerns consumers: a semantic approach to product feature extraction from 
consumer reviews, Information Systems and E-Business Management, 8 (2) 149-167.

[6] Eirinaki, M., Pisal, S., Singh, J. (2012). Feature-based opinion mining and ranking. J. Comput. Syst. Sci., 78, 1175-1184.

[7] Qiu, G., Liu, B., Bu, J., Chen, C. (2011). Opinion word expansion and target extraction through double propagation, Comput. Linguist. 37 (1) 9-27.

[8] Somprasertsri, G., Lalitrojwong, P. (2010). Mining feature-opinion in online customer eviews for opinion summarization, J. Univers. Comput. Sci. 16 (6) 938-955.

[9] Wang, G. A., Jiao, J., Abrahams, A. S., Fan, W., Zhang, Z. (2013). Expertrank:, A topic-aware expert finding algorithm for online knowledge communities, Decis. Support Syst. 54 (3) 1442-1451.

[10] Golub, G. H., Van Loan, C. F. (2012). Matrix Computations, Johns Hopkins University Press, Baltimore, MD, 2012.

[11] Titov, I., McDonald, R. (2008). A joint model of text and aspect ratings for sentiment summarization, in: Proceedings of the Annual Meeting on Association for Computational Linguistics and the Human Language Technology Conference (ACL-HLT), Publishing, p. 308-316.

[12] IRESEARCH, China internet shopping market report in 2013, 2014-5, <http://ec.iresearch.cn/shopping/ 20140114/224908.shtml>

[13] Toutanova, K., Klein, D., Manning, C. D., Singer, Y. (2003). Feature-rich part-of-speech tagging with a cyclic dependency network, in: Proceedings of HLT-NAACL, Publishing, p. 252-259.

[14] Kang, Y., Zhou, L. (2017). RubE: Rule-based methods for extracting product features from online consumer reviews. Inf. Manag. 54, 166-176.

[15] Li, S., Zhou, L., Li, Y. (2015). Improving aspect extraction by augmenting a frequency-based method with web-based similarity measures. Inf. Process. Manag. 51, 58-67.

[16] Long, C., Zhang, J., Zhut, X. (2010). A Review Selection Approach for Accurate Feature Rating Estimation. In: Proceedings of the 23rd International Conference on Computational Linguistics: Posters, Beijing, China, 2327 August 2010; Association for Computational Linguistics: Stroudsburg, PA, USA, p. 766-774.

[17] Aljukhadar, M., Senecal, S., Daoust, C. E. (2012). Using recommendation agents to cope with information overload, Int. J. Electron. Commer. 17 (2) 41-70.

[18] Hu, M., Liu, B. (2004). Mining and summarizing customer reviews, in: Proceedings of International Conference on Knowledge Discovery and Data Mining, Publishing, ACM SIGKDD, p. 168-177.

[19] Hu, M., Liu, B. (2004). Mining opinion features in customer reviews, In: Proceedings of 19th National Conference on Artificial Intelligence, Publishing, AAAI Press, p. 755-760.

[20] Popescu, A. M., Etzioni, O. (2005). OPINE: Extracting Product Features and Opinions from Reviews. In: Proceedings of the HLT/EMNLP on Interactive Demonstrations, Vancouver, BC, Canada, 7 October 2005; Association for Computational Linguistics: Stroudsburg, PA, USA, p. 32-33.

[21] Poria, S., Ofek, N., Gelbukh, A., Hussain, A., Rokach, L. (2014). Dependency tree-Based rules for concept-level aspect-based sentiment analysis. In Semantic Web Evaluation Challenge; Springer: Cham, Switzerland, p. 41-47.

[22] Su, Q., Xiang, K., Wang, H., Sun, B., Yu, S. (2006). Using pointwise mutual information to identify implicit features in customer reviews, In: Proceedings of 17 th International Conference on Computer Processing of Oriental Languages, Publishing, p. 22-30.

[23] Qiu, G., Liu, B., Bu, J., Chen, C. (2009). Expanding domain sentiment lexicon through double propagation. In: Proceedings of the 21st International Jont Conference on Artifical Intelligence, Pasadena, CA, USA, 11-17 July 2009; Morgan Kaufmann Publishers Inc.: San Francisco, CA, USA, p. 1199-1204.

[24] Lau, R.Y., Li, C., Liao, S. S. (2014). Social analytics: learning fuzzy product ontologies for aspect-oriented sentiment analysis, Decis. Support Syst. 65, p. 80-94.

[25] Rana, T. A., Cheah, Y. N. (2017). A Two-Fold RuleBased Model for Aspect Extraction. Expert Syst. Appl. 89, 273-285.

[26] Brin, S., Page, L. (1998). The anatomy of a largescale hypertextual web search engine, Comput. Netw. ISDN Syst. 30 (1) 107-117.

[27] Brody, S., Elhadad, N. (2010). An unsupervised aspect-sentiment model for online reviews, In: Proceedings of Annual Conference of the North American Chapter of the Association for Computational Linguistics, Publishing, Association for Computational Linguistics, p. 804812.

[28] Scaffidi, C., Bierhoff, K., Chang, E., Felker, M., Ng, H., Jin, C. (2007). Red Opal: Product-feature scoring from reviews. In: Proceedings of the 8th ACM Conference on Electronic Commerce, San Diego, CA, USA, 11-15 June 2007; ACM: New York, NY, USA, p. 182-191.

[29] Su, S. H., Lynn, K. T. (2013). Extracting Product Features and Opinion Words Using Pattern Knowledge in Customer Reviews. Sci. World J. 2013, 394758:1394758:5.

[30] Thet, T. T., Na, J. C., Khoo, C. S. (2010). Aspectbased sentiment analysis of movie reviews on discussion boards, Journal of Information Science, 36 (6) 823-848.

[31] Fan, W. G., Gordon, M. D. (2014). The power of social media analytics, Commun. ACM 57 (6), p. 74-81.

[32] Fu, X., Liu, G., Guo, Y., Wang, Z. (2013). Multi-aspect sentiment analysis for Chinese online social reviews based on topic modeling and HowNet lexicon, Knowledge- 
Based Systems, 37, 186-195.

[33] Wu, Y., Zhang, Q., Huang, X., Wu, L. (2009). Phrase dependency parsing for opinion mining, In: Proceedings of the 2009 Conference on Empirical Methods in Natural Language Processing, (Singapore), p. 1533-1541.

[34] Chen, Y. -C., Shang, R. -A., Kao, C. -Y. (2009). The effects of information overload on consumers subjective state towards buying decision in the internet shopping environment, Electron. Commer. Res. Appl. 8 (1) 48-58.

[35] Yan, Z., Xing, M., Zhang, D., Ma, B. (2015). EXPRS: An extended pagerank method for product feature extraction from online consumer reviews. Inf. Manag. 52, 850858.

[36] Zhai, Z., Liu, B., Xu, H., Jia, P. (2011). Constrained LDA for grouping product features in opinion mining, In: Proceedings of 15th Pacific-Asia Conference, Advances in Knowledge Discovery and Data Mining, p. 448-459.

[37] Zhang, L., Liu, B., Lim, S. H., O Brien-Strain, E. (2010). Extracting and ranking product features in opinion documents. In: Proceedings of the 23rd International Conference on Computational Linguistics: Posters, Beijing, China, 23-27 August 2010; Association for Computational
Linguistics: Stroudsburg, PA, USA, p. 1462-1470.

[38] Zhuang, L., Jing, F., Zhu, X. Y. (2006). Movie review mining and summarization. In: Proceedings of the 15th ACM International Conference on Information and Knowledge Management, Arlington, VA, USA, 06-11 November 2006; ACM: New York, NY, USA, p. 43-50.

[39] Poria, Soujanya., Cambria, Erik., Ku, Lun-Wei., Gui, Chen., Gelbukh, Alexander. (2014). A Rule-Based Approach to Aspect Extraction from Product Reviews. In: Proceedings of the Second Workshop on Natural Language Processing for Social Media, Dublin, Ireland, August p. 28-37.

[40] Rana, T. A., Cheah, Y. N. (2015). Hybrid rule-based approach for aspect extraction and categorization from customer reviews. In: Proceedings of 9th International Conference on IT in Asia (CITA), Kota Samarahan, Malaysia, (August) p. 1-5.

[41] Ansari, Gunjan., Saxena, Chandni., Ahmad, Tanvir., Doja, M. N. (2020). Aspect Term Extraction using Graphbased Semi-Supervised Learning, In: Proceedings of International Conference on Computational Intelligence and Data Science (ICCIDS 2019), (February) p. 2080-2090. 